Tropical Journal of Pharmaceutical Research October 2012; 11 (5): 823-833

(C) Pharmacotherapy Group,

Faculty of Pharmacy, University of Benin

Benin City, 300001 Nigeria.

All rights reserved.

Available online at http://www.tjpr.org

Research Article

http://dx.doi.org/10.4314/tjpr.v11i5.17

\title{
Caesarean Risk Factors in Northern Region of Bangladesh: A Statistical Analysis
}

\author{
Mostafizur Rahman ${ }^{1}$, Asma Ahmad Shariff ${ }^{1^{*}}$ and Aziz Shafie ${ }^{2}$ \\ ${ }^{1}$ Centre for Foundation Studies in Science, ${ }^{2}$ Department of Geography, University of Malaya, Kuala Lumpur, Malaysia
}

\begin{abstract}
Purpose: To explore the measurement of a scale of caesarean (C-section) risk factors and degree of risk contribution in different health facilities and to determine a suitable graphical representation (image) of caesarean cases.

Methods: Based on seventeen indicators, a composite index was computed for each respondent and classified into three groups using Beta distribution of first kind. For the analysis of contribution of risk factors between private and public patients, principal component analysis (PCA) was also used. An attempt was made to visualise a suitable graphical representation of caesarean cases by independent component analysis (ICA).

Results: The selected risk factors were more contributory to public hospital patients than to those in private hospitals on the basis of higher estimated value of range $(R=0.134)$ but a higher proportion of C-section occurred in private (93.4\%) than in public hospitals (30.3\%). On the other hand, PCA showed that the contribution of selected risk factors accounts for approximately $60.0 \%$ and $68.5 \%$ in private and public hospitals, respectively. Furthermore, from the various graphical representation, the numbers of private patients were more interlinked by ICA but not of the other graphical representations of PCA.

Conclusion: We had expected the rate of C-section would be higher among public hospital patients than private hospital patients but the results obtained indicate the reverse. It seems that the combination of the propensity of private practice doctors to carry out $C$-section and the financial benefits on the part of private hospitals may be contributory factors to the caesarean section rates in private health facilities.
\end{abstract}

Keywords: Caesarean risk factors, Composite index, Principal component analysis (PCA), Independent component analysis (ICA). 


\section{INTRODUCTION}

Deliveries normally occur in one of two ways - normal or caesarean section (C-section). Prior to 1970, C-section was an uncommon procedure in Bangladesh, as less than one percent of the deliveries were by caesarean. During the period 2003-2004, based on Bangladesh Demographic Health Survey (BDHS), mean C-section rate was only 2.5 $\%$. As of $2010,12.2 \%$ of births were delivered by $\mathrm{C}$-section [1]. Since then, Csection has become more common in Bangladesh like in most other comparable countries [2-4].

Expanded fetal indications, law and rules concerning medical liability and development of new medical technology are examples of factors believed to be responsible for the rapid increase in C-section in the late 1970s. Moreover, numerous factors such as geographical region, physicians' practice styles, maternal and socio-demographic characteristics are also associated with variation by place of delivery $[5,6]$. Several studies in different countries found that the rate of caesarean delivery in private hospitals was higher than in public hospitals [7-9].

In previous studies which examined the risk factors of caesarean delivery either in private or public hospitals, various methods were described including logistic regression [10], decision tree analysis [11], neural networks [12] and a simple and robust method [13]. No research has been conducted measures the scale of risk factors and variation in those factors that may have influence on the rate of caesarean delivery. Therefore, the specific objectives of this paper are: (i) to estimate the scale of risk factors from caesarean cases by considering the composite index of risk factor, (ii) to isolate those risk factors which contribute to the increase in the rate of caesarean deliveries, and (iii) to determine a suitable graphical representation (image) of caesarean cases from different locations on the scale of component score.

\section{EXPERIMENTAL}

\section{Study area}

The study sample comprised of 1142 women who had either caesarean or non-caesarean delivery at 4 private and 4 public hospital maternity wards in the northern region of Bangladesh. Among the 1142 delivery cases, 652 were caesarean and the remaining 490 were non-caesarean.

\section{Sampling design}

The study carried out followed a crosssectional design where data were collected by direct interviews. The participants were selected by simple random sampling. Most of the questions were close-ended and the answers chosen by the respondents were indicated by tick mark.

\section{Selection of risk factors and their measurements}

Webster et al [14] suggested that women with medical complications near delivery are more likely to undergo C-section in order to improve their survival prospects as well as their newborns. Therefore, maternal factors (clinical) were considered as risk factors in the present study. Maternal factors considered include prolonged labour (> $12 \mathrm{~h}$ ), fetal distress, previous C-section, sensationless and swollen leg, breathing difficulty, child aborted around delivery time, multiple births, head circumference of newborns, and length and weight of babies. Previous works also suggested that a thorough understanding of decisions to perform C-section should recognize many non-medical factors as playing important part in such decisions [15]. In order to investigate the non-medical determinants of C-section, a set of sociodemographic factors such as maternal age at birth, age at marriage, birth order, and mother's educational level was identified. Padmadas et al [16] and Misra et al [17] have also found that there is a strong association between $\mathrm{C}$-section and place of residence. 
Table 1: Selected risk factors and their measurement

\begin{tabular}{|c|c|c|c|}
\hline Risk factor & Measurement & Risk factor & Measurement \\
\hline Mother's Education (ME) & $\begin{array}{l}\text { 1= Primary and below } \\
2=\text { Secondary, } 3= \\
\text { Higher }\end{array}$ & $\begin{array}{l}\text { Prolonged Labour } \\
\text { (more than 12h) (LTL) }\end{array}$ & $(0=$ No, $1=$ Yes $)$ \\
\hline Fetal Distress (FD) & $(0=$ No, $1=$ Yes $)$ & Residence (R_C) & $\begin{array}{l}1=\text { Rural, } 2= \\
\text { Urban }\end{array}$ \\
\hline Previous C-Section (AO) & $(0=$ No, $1=$ Yes $)$ & $\begin{array}{l}\text { Mother's Age: years } \\
\text { (MA_C) }\end{array}$ & $\begin{array}{l}1=<20,2=20-24 \\
3=25-29,4=30+\end{array}$ \\
\hline $\begin{array}{l}\text { Pregnancy Induced Senseless } \\
\text { (PIS) }\end{array}$ & $(0=$ No, $1=$ Yes $)$ & $\begin{array}{l}\text { Age at Marriage: years } \\
\text { (AM_C) }\end{array}$ & $\begin{array}{l}1=<18,2=18-22 \\
3=23+\end{array}$ \\
\hline $\begin{array}{l}\text { Pregnancy-Induced Swollen Leg } \\
\text { (PISL) }\end{array}$ & $(0=$ No, $1=$ Yes $)$ & $\begin{array}{l}\text { Length of Baby: } \mathrm{cm} \\
\text { (HB_C) }\end{array}$ & $1=<45,2=45+$ \\
\hline Multiple Birth (MB) & $(0=$ No, $1=$ Yes $)$ & $\begin{array}{l}\text { Head Circumferences: } \\
\text { cm (HC_C) }\end{array}$ & $1=<32,2=32+$ \\
\hline Ever had a Child Aborted (ECA) & $(0=$ No, $1=$ Yes $)$ & $\begin{array}{l}\text { Weight of Baby: kg } \\
\text { (WB_NC) }\end{array}$ & $1=<2.5,2=2.5+$ \\
\hline $\begin{array}{l}\text { Pregnancy-Induced Breathing } \\
\text { Difficulty (PIBD) }\end{array}$ & $(0=$ No, $1=$ Yes $)$ & Ordēer of Birth (BODER) & $1=1,2=2,3=3+$ \\
\hline $\begin{array}{l}\text { Duration of Taking Balanced } \\
\text { Diet (DTN) }\end{array}$ & \multicolumn{3}{|c|}{ 1= Often, $2=$ Once a week, 3= Rarely } \\
\hline
\end{tabular}

Furthermore, numerous socio-economic and cultural factors influence the decision on pattern of feeding and balance diet that may also influence delivery. Thus, place of residence and the duration of the period balance diet was taken were also considered as risk factors in the analysis. Thus, the selected risk factors and their measurement are shown in Table 1.

\section{Hypothesis of setting}

Since the rate of caesarean delivery is higher in private hospitals than in public hospitals, this study assessed the following hypotheses on place of delivery for the woman who had a caesarean delivery (CD).

(a)H1: Higher rate of CD imply higher number of risk factors, which contribute to the likelihood of the woman having a C-section. Conversely, lower rate of CD means lower number of risk factors, which translates to the woman being less likely to have $C D$.

(b)H2: The more the interlinks among risk factors of $C D$ the higher the rate of C-section, while the fewer the interlinks among risk factors the lower the rate of $\mathrm{C}$-section.

Necessary steps for composite index of risk factors and its scale measurement Step 1: Standardized the risk factors

$Z_{\mathrm{ij}}=\frac{X_{\mathrm{ij}}-\overline{\mathrm{B}_{j}}}{\mathrm{y}_{\mathrm{j}}}$

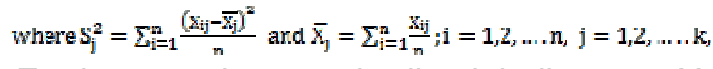
$Z_{\mathrm{ij}}$ denotes the standardized indicators, $X_{i j}$ represent the ith respondent of jth risk, $\bar{X}_{j}$ and $S_{j}$ are the mean and standard deviation of $X_{i j}$ respectively.

\section{Step 2: Compute the composite risk factors}

First, the highest standardized value of respondent for each indicator (with maximum/minimum standardized value depending upon the direction of the indicator) is identified and from this, the deviations of the value for each respondent are taken for all indicators as in Eq 2.

$c_{i}=\left\{\sum_{j=1}^{k}\left(z_{i j}-z_{0 j}\right)^{2}\right\}^{\frac{1}{2}}$ 
where $Z_{0 j}$ is the standardized value of the $j$-th indicator of those respondents having the highest standardized value and $C_{i}$ denote the pattern of composite risk factors of i-th respondent. Secondly, the successive way is used to calculate the index of risk factors:

$R_{1}=\frac{c_{i}}{c}$, where $\mathrm{c}=\overline{\mathrm{C}}+2 \mathrm{~S}, \overline{\mathrm{C}}=$

$\sum_{1=1}^{n} \frac{\Sigma_{1}}{n}$ and $s=\sum_{i=1}^{n} \frac{\left(c_{1} \bar{c}\right)^{2}}{\pi}$

From the value of $R_{i}$, the value of composite index is non-negative and lies between 0 and 1. Hence, we assumed that the mean of $R_{i}$ has a Beta distribution in the range $(0,1)$. Therefore, let the form of the distribution be as in Eq 3.

$f(x)=\frac{1}{Z\left(\mathrm{cu}_{0} \beta\right)} \mathrm{r}^{\mathrm{k}-1}(1-r)^{\beta-1}$ for $\alpha<r<1, \alpha>0$ and $\beta>0$

Step 3: Consider the mean and variance of Beta distribution from Eq 3.

$\operatorname{Mean}(\overline{\mathrm{r}})=\frac{\alpha}{\alpha+\beta}$

Variance $\left(\alpha_{p}^{2}\right)=\frac{\alpha \beta}{(a+\beta)^{2}(a+\beta+1)}$

\section{Step 4: Estimate the parameters}

The values of $\overline{\mathrm{r}}$ and $\sigma_{\mathrm{r}}^{2}$ from $\mathrm{R}_{\mathrm{i}}$, and the parameters $\alpha$ and $\beta$, were calculated, and then estimated from Eqs 4 and 5.

\section{Step 5: Scale measurement}

Let the range of $(0,1)$ be divided into three categories $\left(0, Z_{1}\right),\left(Z_{1}, Z_{2}\right),\left(Z_{2}, 1\right)$, all being linear intervals such that each interval has the same probability weight of 0.33 . Thereafter, the value of $Z_{1}$ and $Z_{2}$ were calculated from a table of the probability density function of Beta distribution.

\section{Data analysis}

Based on the selected risk factors, a composite index of risk factors of some necessary steps was constructed. The procedure for the analysis carried out is as follows. Using Beta distribution of first kind in the range $(0,1)$, the composite index of risk factors was divided into three groups, that is, category I, II and III and are referred to as high, medium and low impact risk factors, respectively. To measure the scale of the composite index, a descriptive statistic, mainly the range $(R)$ is used. In order to explain the extent of total variation in the empirical data, a multivariate technique known as Principal Component Analysis (PCA) was adopted [18]. Finally, a recently developed method known as Independent Component Analysis (ICA) [19] was used to obtain a suitable graphical representation for the image of caesarean cases by place of delivery on component score. Application of both analytical techniques (PCA and ICA) are as detailed by Jolliffe [18] and Aapo et al [19]. The data were analysed using the Statistical Packages for Social Sciences (SPSS) for Windows (version 17.0), R packages (2009) and Microsoft Office Excel (2007).

\section{RESULTS}

Based on 17 indicators, the classifying composite indices calculated for each respondent and the values of range on indices are presented in Table 2. For classification purpose, at private hospital level, we divided the composite index into three class intervals $(0,0.108),(0.108,0.174)$ and $(0.174,1)$ using the Beta distribution of first kind in the range $(0,1)$. Similarly, we obtained three intervals, which are $(0,0.134)$, $(0.134,0.204)$ and $(0.204,1)$, for classification purpose at public hospital level. For relative comparison, the classifying composite indices were placed in categories I, II and III, represented as high, medium and low impact risk factor, respectively. Based on the result noted for the value of range $(R)$, it indicated that there was no big difference between the medium and low risk factor category at private and public hospital level. However, for category I, R $=0.134$, which is higher in public hospitals than in private 
hospitals. Thus, based on the results of $R$, it is interesting to note that the empirical risk factors contributed more to caesarean birth rate in public hospitals than in private hospitals. Interestingly, the result obtained in this study through the survey shows the reverse, i.e., private hospitals, $93.4 \%$ vs public hospitals, $30.3 \%$. To achieve a more complete assessment of the risk factors and their level of contribution to C-section among both private and public hospital patients,

Table 2: Classification of composite index by place of delivery

\begin{tabular}{|c|c|c|c|c|}
\hline \multirow[t]{2}{*}{ Category } & \multicolumn{2}{|c|}{ Private hospital } & \multicolumn{2}{|c|}{ Public hospital } \\
\hline & $\begin{array}{l}\text { Class } \\
\text { interval }\end{array}$ & Range (R) & $\begin{array}{c}\text { Class } \\
\text { interval }\end{array}$ & Range $(R)$ \\
\hline I: High impact risk factor & $0-0.108$ & 0.108 & $0-0.134$ & 0.134 \\
\hline II: Medium impact risk factor & $0.108-0.174$ & 0.066 & $0.134-0.204$ & 0.070 \\
\hline III: Low impact risk factor & $0.174-1$ & 0.826 & 0.204-1 & 0.796 \\
\hline Number of caesarean cases $(n)$ & \multicolumn{2}{|c|}{$453 / 485[93.4 \%]$} & \multicolumn{2}{|c|}{$199 / 657[30.3 \%]$} \\
\hline Mean(r) & \multicolumn{2}{|c|}{0.150} & \multicolumn{2}{|c|}{0.179} \\
\hline Variance $\left(\sigma_{\mathrm{r}}^{2}\right)$ & \multicolumn{2}{|c|}{0.006} & \multicolumn{2}{|c|}{0.007} \\
\hline \multicolumn{5}{|l|}{ Estimated parameter } \\
\hline$\alpha$ & \multicolumn{2}{|c|}{03.0375} & \multicolumn{2}{|c|}{03.5862} \\
\hline$\beta$ & \multicolumn{2}{|c|}{17.2125} & \multicolumn{2}{|c|}{16.4262} \\
\hline
\end{tabular}

Table 3: Eigen values and accounted-for 'variance' of risk factors explained by place of delivery

\begin{tabular}{ccccccc}
\hline Component & \multicolumn{5}{c}{ Private hospital } & \multicolumn{3}{c}{$\begin{array}{c}\text { Public hospital } \\
\text { Eigen } \\
\text { value }\end{array}$} & $\begin{array}{l}\text { Percentage of } \\
\text { variance } \\
\text { explained }\end{array}$ & $\begin{array}{l}\text { Cumm. \% of } \\
\text { total } \\
\text { variance }\end{array}$ & $\begin{array}{l}\text { Eigen } \\
\text { value } \\
\text { variance } \\
\text { explained }\end{array}$ & $\begin{array}{l}\text { Cumm. } \\
\text { \% of total } \\
\text { variance } \\
1\end{array}$ & 2.082 & 12.249 & 12.249 & 2.070 & 12.174 & 12.174 \\
2 & 1.951 & 11.477 & 23.726 & 1.903 & 11.192 & 23.367 \\
3 & 1.379 & 8.110 & 31.836 & 1.610 & 9.472 & 32.839 \\
4 & 1.320 & 7.762 & 39.598 & 1.404 & 8.258 & 41.097 \\
5 & 1.224 & 7.202 & 46.800 & 1.342 & 7.896 & 48.993 \\
6 & 1.125 & 6.617 & 53.417 & 1.187 & 6.981 & 55.974 \\
7 & 1.115 & 6.558 & 59.975 & 1.097 & 6.451 & 62.425 \\
8 & 0.998 & 5.872 & 65.847 & 1.026 & 6.033 & 68.458 \\
9 & 0.922 & 5.421 & 71.268 & 0.907 & 5.336 & 73.794 \\
10 & 0.850 & 4.998 & 76.266 & 0.825 & 4.853 & 78.647 \\
11 & 0.758 & 4.461 & 80.727 & 0.764 & 4.497 & 83.144 \\
12 & 0.745 & 4.381 & 85.108 & 0.701 & 4.125 & 87.269 \\
13 & 0.693 & 4.074 & 89.182 & 0.627 & 3.690 & 90.959 \\
14 & 0.664 & 3.905 & 93.087 & 0.534 & 3.144 & 94.102 \\
15 & 0.512 & 3.011 & 96.098 & 0.414 & 2.436 & 96.538 \\
16 & 0.500 & 2.939 & 99.038 & 0.390 & 2.292 & 98.830 \\
17 & 0.164 & 0.962 & 100.000 & 0.199 & 1.170 & 100.000 \\
\hline
\end{tabular}


Rahman et al

Table 4: Component loading by selected risk factors for C-section: Private vs. Public hospital

\begin{tabular}{|c|c|c|c|c|c|c|c|c|}
\hline \multirow{2}{*}{ Risk factor } & \multicolumn{8}{|c|}{ Component } \\
\hline & 1 & 2 & 3 & 4 & 5 & 6 & 7 & 8 \\
\hline \multicolumn{9}{|c|}{ Private hospital } \\
\hline $\mathrm{ME}$ & .725 & -.257 & .078 & .020 & .038 & .043 & .098 & - \\
\hline FD & -.273 & .074 & .014 & -.326 & -.162 & .502 & .227 & - \\
\hline $\mathrm{AO}$ & -.158 & .448 & .143 & .077 & .594 & .042 & -.235 & - \\
\hline PIS & .008 & .015 & .053 & .412 & -.104 & .589 & .176 & - \\
\hline PISL & .014 & -.045 & -.034 & .365 & .567 & .096 & .394 & - \\
\hline MB & -.098 & .288 & .061 & .581 & -.204 & .103 & -.316 & - \\
\hline ECA & -.023 & .124 & .034 & -.125 & .763 & -.018 & -.040 & - \\
\hline PIBD & -.011 & -.089 & -.015 & $\underline{.693}$ & .057 & -.052 & .021 & - \\
\hline LTL & -.052 & -.039 & -.045 & .079 & .004 & .000 & -.721 & - \\
\hline R_C & $\underline{-.562}$ & -.059 & -.097 & .102 & .075 & -.097 & .324 & - \\
\hline DTNN & .220 & .045 & .068 & -.043 & .164 & .619 & -.212 & - \\
\hline MA_C & .424 & .737 & -.051 & -.029 & -.041 & .045 & .164 & - \\
\hline AM_C & .845 & $\overline{.075}$ & -.049 & .010 & -.023 & -.031 & .127 & - \\
\hline HB_C & -.006 & .267 & .419 & .052 & -.296 & -.389 & .189 & - \\
\hline $\mathrm{HC} \_\mathrm{C}$ & -.096 & .097 & .766 & -.048 & .001 & .139 & .103 & - \\
\hline WB_NC & .197 & -.146 & .703 & .056 & .084 & -.032 & -.123 & - \\
\hline BODĒER & -.154 & .870 & $-\overline{-.030}$ & -.028 & .050 & .006 & .037 & - \\
\hline \multicolumn{9}{|c|}{ Public hospital } \\
\hline $\mathrm{ME}$ & -.187 & .785 & .058 & .051 & -.013 & -.166 & .106 & .081 \\
\hline FD & .004 & $-\overline{-.295}$ & .287 & -.018 & .276 & .598 & .035 & -.084 \\
\hline $\mathrm{AO}$ & .163 & -.062 & -.055 & -.032 & .022 & .134 & .786 & .094 \\
\hline PIS & .007 & .082 & -.113 & -.043 & -.083 & .784 & .113 & -.025 \\
\hline PISL & -.180 & .108 & -.032 & .252 & .636 & .053 & .276 & -.088 \\
\hline MB & .391 & -.030 & -.280 & .339 & .328 & .120 & -.386 & .240 \\
\hline ECA & .121 & -.013 & -.023 & -.186 & .753 & -.021 & -.152 & .000 \\
\hline PIBD & .360 & -.020 & -.272 & .622 & $\overline{-.001}$ & -.016 & .208 & .148 \\
\hline LTL & -.143 & .033 & .172 & .828 & -.041 & -.079 & -.138 & -.116 \\
\hline R_C & -.098 & -.402 & -.190 & $-\overline{-.165}$ & .249 & -.213 & -.258 & .229 \\
\hline DTN & -.031 & .079 & -.008 & -.008 & -.027 & .099 & -.118 & -.833 \\
\hline MA_C & .838 & .332 & .114 & .005 & .003 & .145 & .006 & .030 \\
\hline AM_C & .242 & .791 & -.038 & -.083 & .150 & .048 & -.269 & -.058 \\
\hline HB_C & -.038 & .225 & .420 & -.060 & -.223 & .095 & -.137 & .550 \\
\hline $\mathrm{HC} \_\mathrm{C}$ & .117 & -.045 & .800 & -.023 & -.080 & .074 & -.091 & .123 \\
\hline WB_NC & .074 & .224 & .588 & .148 & .141 & -.391 & .318 & -.070 \\
\hline BODER & .806 & -.269 & .110 & -.006 & -.019 & -.154 & .203 & -.046 \\
\hline
\end{tabular}

principle component analysis (PCA) method was used. The main features, which are outlined in Tables 3 and 4, are essentially as follows.

\section{Private hospitals}

Based on Table 3, it was found that seven components account for about $60 \%$ of the total accounted-for variance, and their component loading are displayed in Table 4. 
Each risk factor's highest (absolute) loading is underlined in the table. From the component loadings, it was seen that component 1 , which accounted for more than $12 \%$ of the total variance, is clearly dominated by ME, R_C and AM_C. These are socio-demographic impact factors. Component 2, which accounted for more than $23 \%$ of the total variance, is dominated by MA_C and BODER. These components appear to reflect demographic factors. The risk factors loading on components 3 and 4 are represented by double risk factors (HC_C \& WB_NC) and (MB \& PIBD ), while components 5 and 6 are triple risk factors (AO, PISL \& ECA) and (FD, PIS, \& DTN); they accounted for about 32, 40, 47 and $53.42 \%$ of the total variance, respectively. The remaining component, represented by a single risk factor $L T L$, was highest load on component 7 .

\section{Public hospitals}

Table 3 also shows that the first eight components made up $68.5 \%$ of the total variability in the empirical data. It is clear from the data in Tables 3 and 4 that the first component explained more than $12 \%$ of the total variance. This component is high and shows positive loading with MA_C and BODER. Consequently, component one might be interpreted as measuring a demographic impact factor. More than $23 \%$ of the total variance was accounted for by component 2 and showed highly positive loading with $\mathrm{ME}$ and AM_C. Thus, the second component might be interpreted as measuring socio-demographic impact factors. Components 3, 4, 5, 6 and 8 accounted for $32,41,48,55$ and $68 \%$ of the total variance, each corresponding to, and represented by double risk factors (HC_C \& WB_NC), (PIBD \& LTL), (PISL \& ECA), (FD \& PIS) and (DTN \& HB_C), respectively. Only $56.0 \%$ is accounted for component 7 which showed a highly positive loading with $\mathrm{AO}$. From a statistical point of view, this analysis clearly shows that considering level of contribution obtained from components 1 and 2, the impacts of the risk factors are about the same for both places of delivery. Furthermore, a higher level of contribution was found in the remaining components in public hospitals than in private hospitals. Therefore, in our interpretation, we have expected the rate of $\mathrm{C}$-section would be higher among public hospital patients than among private hospital patients, but the results obtained showed the reverse, and hence, our first hypothesis $(\mathrm{H} 1)$ was not buttressed by the actual results obtained.

This study also focused on graphical plots namely, biplot, three dimensional scatter plot and loading plot, in order to visualize the principal components in the multivariate data. As shown in Fig 1, the direction and length of the vectors indicate how each factor (here, V1 refers to ME, V2 refers to FD and others consecutively refers to the other factors) contributes towards the two principal components in the plot. Fig 1 also shows that first two components only represent more than $23.7 \%$ of cumulative variance among private hospital patients, followed by public hospital patients $(\geq 23.3 \%)$. Alternatively, to visualize the first three principal component coefficients for each factor, the three dimensional biplot by different health facilities are shown in Fig 2. In this analysis, the three dimensional biplot that represents the first three components only accounted for about 32 and $33 \%$ of cumulative variance among private and public hospital patients, respectively. From Fig 2, it was also clear that maternal risk factors are more interlinked to each other in public hospital patients but this was not evident among private hospital patients. We had expected that the rate of $\mathrm{C}$-section will be higher in public hospitals than in private hospitals but this was not so in the case of $\mathrm{C}$-section as seen in private hospitals; hence, our second hypothesis $(\mathrm{H} 2)$ was also not confirmed by the results obtained.

Fig 3 shows the loading plot by place of delivery and also how each factor contributes to the loading of each component. From the plot, one can identify the specific factors of the main components which could not be easily identified in the biplot and three dimensional biplot. Finally, the interrelationship among the caesarean cases could easily be observed by ICA rather than PCA (see Fig 4) as well as biplot, three dimensional biplot and loading plot. 
Rahman et al
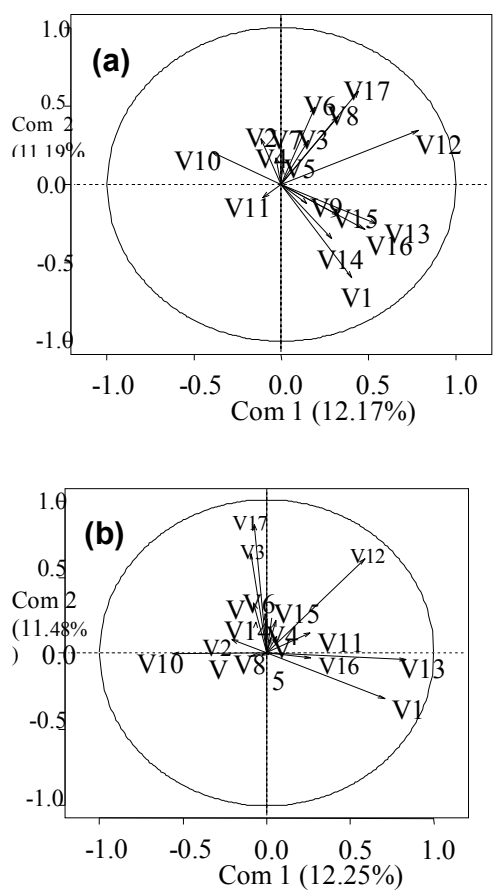

Fig 1: Biplot of (a) private and (b) public hospitals
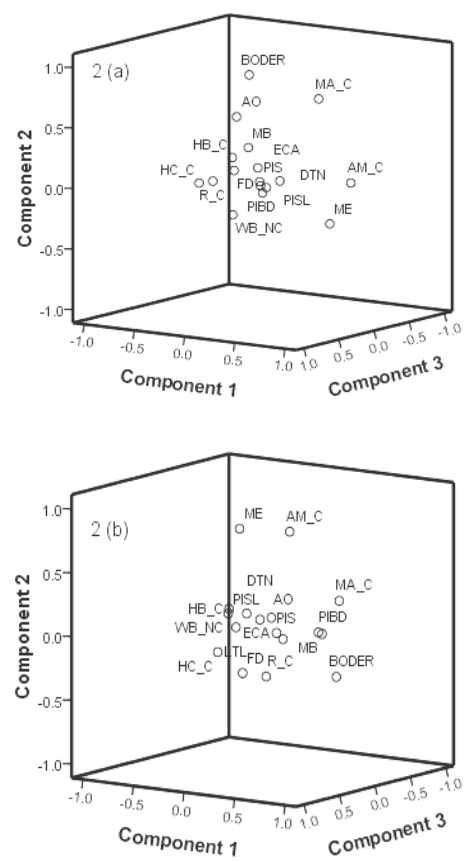

Fig 2: Three dimensional biplot of (a) private and (b) public hospitals
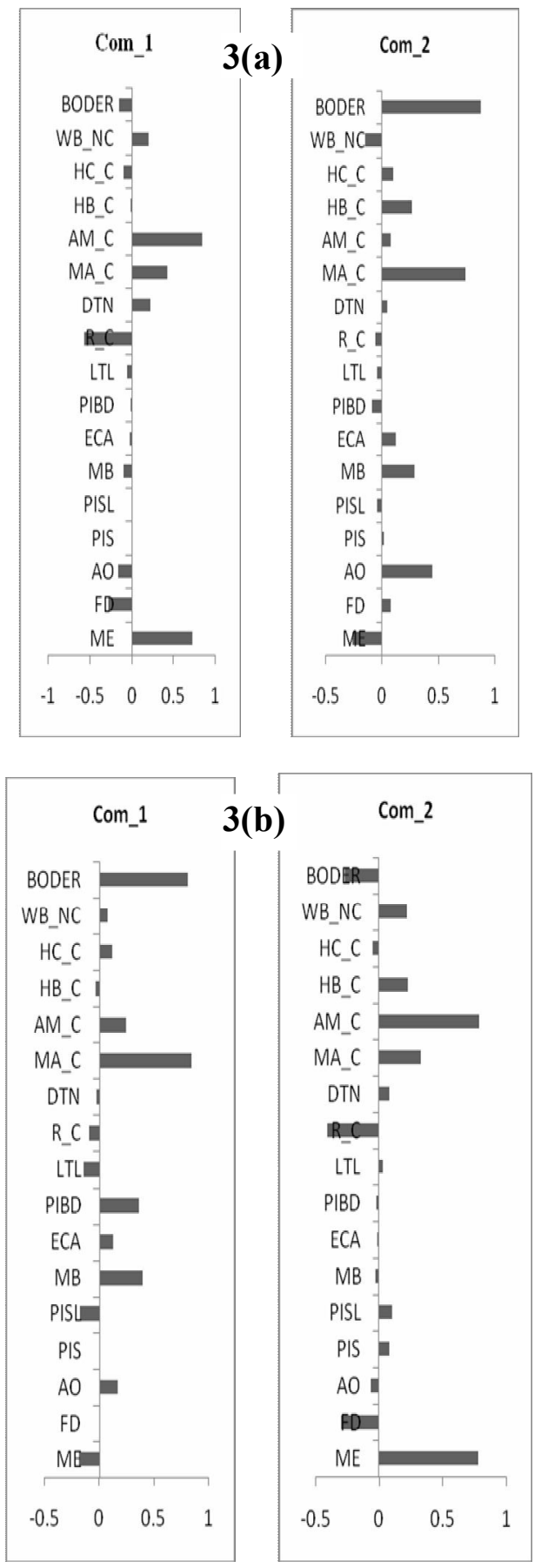

Fig 3: Loading plot of (a) private and (b) public hospitals 

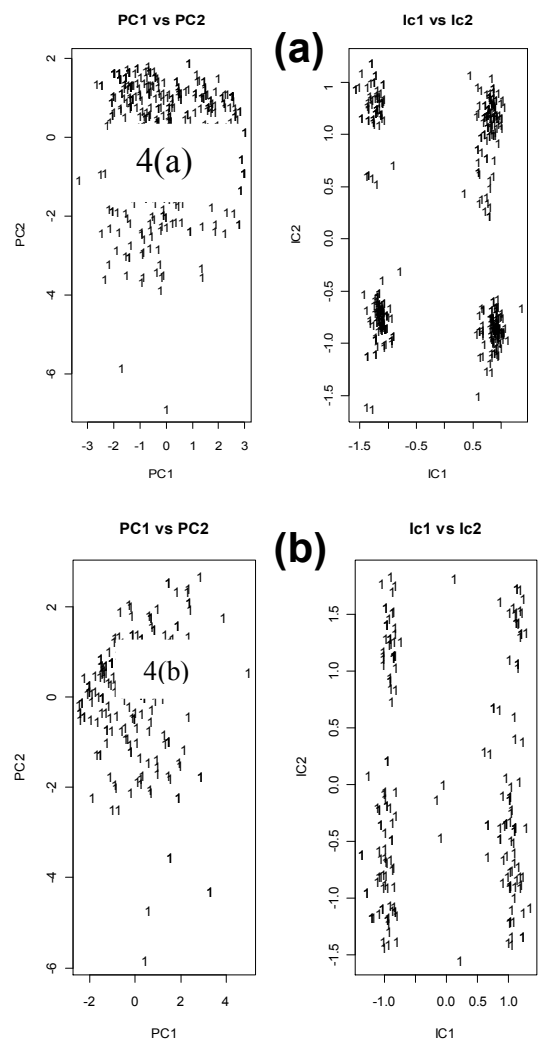

Fig 4: Number of caesarean cases on the scale of component score: (a) private and (b) public hospitals

\section{DISCUSSION}

Almost all the deliveries performed in private hospitals studied in the northern region of Bangladesh were by $\mathrm{C}$-section. It is important to stress at this point that these findings should not be generalized to the northern region or the country as a whole. However, this rate is high compared to that recommended in the literature and by the World Health Organization (WHO) who published guidelines regarding caesarean rates in 1985, revised in 1994. In 1994, WHO stated that no region in the world is justified in having a caesarean rate greater than 10 to $15 \%$ [20].

C-section rates in the different health facilities has been of great concern. The analysis of the $\mathrm{C}$-section deliveries for private and public hospitals substantiates this concern. The rate for private hospitals was higher, where 453 out of 485 births were caesarean deliveries. Previous studies in other countries found that the rate of caesarean delivery in private hospitals was also higher than those in public hospitals [7]. Based on the estimated value of range $(R)$ and PCA in this study, the selected risk factors contributed more to public patients than to private patients. Therefore, as shown in the presented findings, we had expected the rate of $\mathrm{C}$-section to be higher amond public patients than private patients. However, the results obtained in the survey are contrary to our expectations. This may be due to two factors: the practice tendency of private hospital doctors and the financial benefits derived by private hospitals. These factors have also been mentioned in a previous study [21].

Among the risk factors, maternal factors seem to be less important in determining which women would have $\mathrm{C}$-section in private hospitals; however, socio-demographic factors were the most important factors that influenced the decision to perform a Csection. Maternal factors seemed to be more interlinked with each other in public hospitals than in private hospital. Furthermore, by graphical visualization on ICA, the data were more readily observed, and the study found that the numbers of private hospital patients that are more interlinked remain hidden factors that are not shown in public hospital patients. Therefore, it is interesting to note that these hidden factors might come from doctor motivation, doctors' desire for more money, socio-economic status, etc.

\section{Limitations of the study}

First, the findings of this study are only applicable to the northern part of Bangladesh but not the whole of the country. Second, several risk factors have been mentioned in the literature $[5,6]$, all of which were not considered in this study. Another limitation is that although all the women expressed their desire in oral interviews to be involved in 
decisions on delivery option, they however did not feel "medically capable" of making a decision on elective and non-elective Csection. As a result, we could not include maternal choice as a factor in this study. Finally, patients' medical records were not examined to determine on what grounds the doctors opted for caesarean delivery; therefore, we did not look at the physician factor in relation to caesarean birth rates, although other researchers have studied this factor.

\section{CONCLUSION}

From the foregoing analysis, the data suggest unequivocally that the rate of caesarean delivery should be higher in public hospitals. The observed result is the reverse; the rate of caesarean delivery is much higher in private hospitals. Therefore, this study demonstrates that the roles of selected risk factors are not the main source of influence on the rate of caesarean delivery in private hospitals vis a vis public hospitals. Consequently, some other unrecorded factors influence strongly the decision on whether or not to perform a C-section. Since C-section deliveries cost more than vaginal deliveries, both in terms of the number of days of hospitalisation required and the financial costs, it is possible that this very useful surgical procedure is being misused for profit purposes in the private hospitals in the northern region of Bangladesh. Efforts to reduce $C$-section birth in developing countries, like the northern region of Bangladesh, will require a comprehensive approach that would address patients' variables, care giver practices and hospital policies.

\section{REFERENCES}

1. BMMS. Bangladesh maternal mortality and health care survey 2010: Summary of key findings and implications, Dhaka, Bangladesh, 2010; pp 1-12.

2. Menacker F, Curtin SC. Trends in caesarean birth and vaginal birth after previous caesarean,
1991-99. National Vital Statistical Report 2001; 49: 1-16.

3. Martin JA, Park MM, Sutton PD. Births: preliminary data for 2001. National Vital Statistical Report 2002; 50: 1-20.

4. Ravindran J. Rising caesarean section rates in public hospitals in Malaysia 2006. Med J Malaysia 2008; 63(5): 434-435.

5. Acker DB, Haas S, O'Brien C, Donahue CL, Porell $M M$, Sachs BP. Cesarean birth rate: small geographic area analysis. Am J Obsand Gyn 1988; 159: 386-388.

6. Mossialos E, Allin S, Karras K, Davaki K. An investigation of Caesarean section in three Greek hospitals: the impact of financial incentives and convenience. Eur J Pub Health 2005; 15(3): 288-295.

7. Guo S, Padmadas SS, Zhao F, Brown JJ, Stones $R W$. Delivery settings and caesarean section rates in China. Bulletin of World Health Org, Geneva 2007; 85(10): 755-762.

8. Leung GM, Lam TH, Thach TQ, Wan S, Ho LM. Rates of caesarean births in Hong Kong: 1987-1999, Birth 2001; 28: 166-172.

9. Khawaja $M$, Jurdi R, Kabakian T. Rising trends in caesarean section rates in Egypt. Birth 2004; 31(1): 12-16.

10. Hueston WJ. Development of a caesarean delivery risk score. Obs Gyn 1994; 84: 965-968.

11. Sims CJ, Meyn L, Caruana R, Rao RB, Mitchell T, Krohn M. Predicting caesarean delivery with decision tree models. Am J Obsand Gyn 2000; 183: 1198-1206.

12. Macones GA, Hausman N, Edelstein R, Stamilio $D M$, Marder SJ. Predicting outcomes of trials of labor in women attempting vaginal birth after caesarean delivery: a comparison of multivariate methods with neural networks. Am J Obsand Gyn 2001; 184: 409-413.

13. Gordon CSS, Michael D, lan RW, Jill PP. Combined logistic and Bayesian modelling of caesarean section risk. Am J Obsand Gyn 2004; 191: 2029-2034

14. Webster L, Daling J, Mcfarlane C, Ashley D, Warren C. Prevalence and determinants of caesarean section in Jamaica. J Biosoc Sci 1992; 24: 515-525.

15. Gomes UA, Silva AA, Bettiol H, Barbieri MA. Risk factors for the increasing caesarean section rate in Southeast Brazil: a comparison of two birth cohorts, 1978-1979 and 1994. Int J Epidemiol 1999; 28: 687-694.

16. Padmadas $S$, Kumar $S$, Nair $S$, Kumari $A$. Caesarean section delivery in Kerala, India: evidence from a national family health survey. Soc Sci Med 2000; 51: 511-521.

17. Mishra US, Ramanathan M. Delivery-related complications and determinants of caesarean section rates in India. Health Policy Planning 2002; 17: 90-98.

18. Jolliffe IT. Principal Component Analysis. Springer Series in Statistics 2002; 150-166. 


\section{Rahman et al}

19. Aapo H, Oja E. Independent Component Analysis: Algorithms and Application, Neural Networks 2000; 13(4-5): 411-430.

20. WHO. Appropriate technology for birth. Lancet 1985; 2: 436-437.
21. Parkhurst JO, Rahman SA. Life saving or money wasting? Perceptions of caesarean sections among users of services in rural Bangladesh. Health Policy 2007; 80: 392-401. 